The CRDS spectrum of the $a^{1} \Delta_{\mathrm{g}}-X^{3} \Sigma_{\mathrm{g}}^{-}$band of ${ }^{16} \mathrm{O}^{17} \mathrm{O},{ }^{17} \mathrm{O}^{18} \mathrm{O}$ and ${ }^{17} \mathrm{O}_{2}$ has been recorded at $80 \mathrm{~K}$.

The nuclear hyperfine structure of the transitions could be partly resolved.

The hyperfine parameter coupling in the $a^{1} \Delta_{\mathrm{g}}$ state has been derived. 


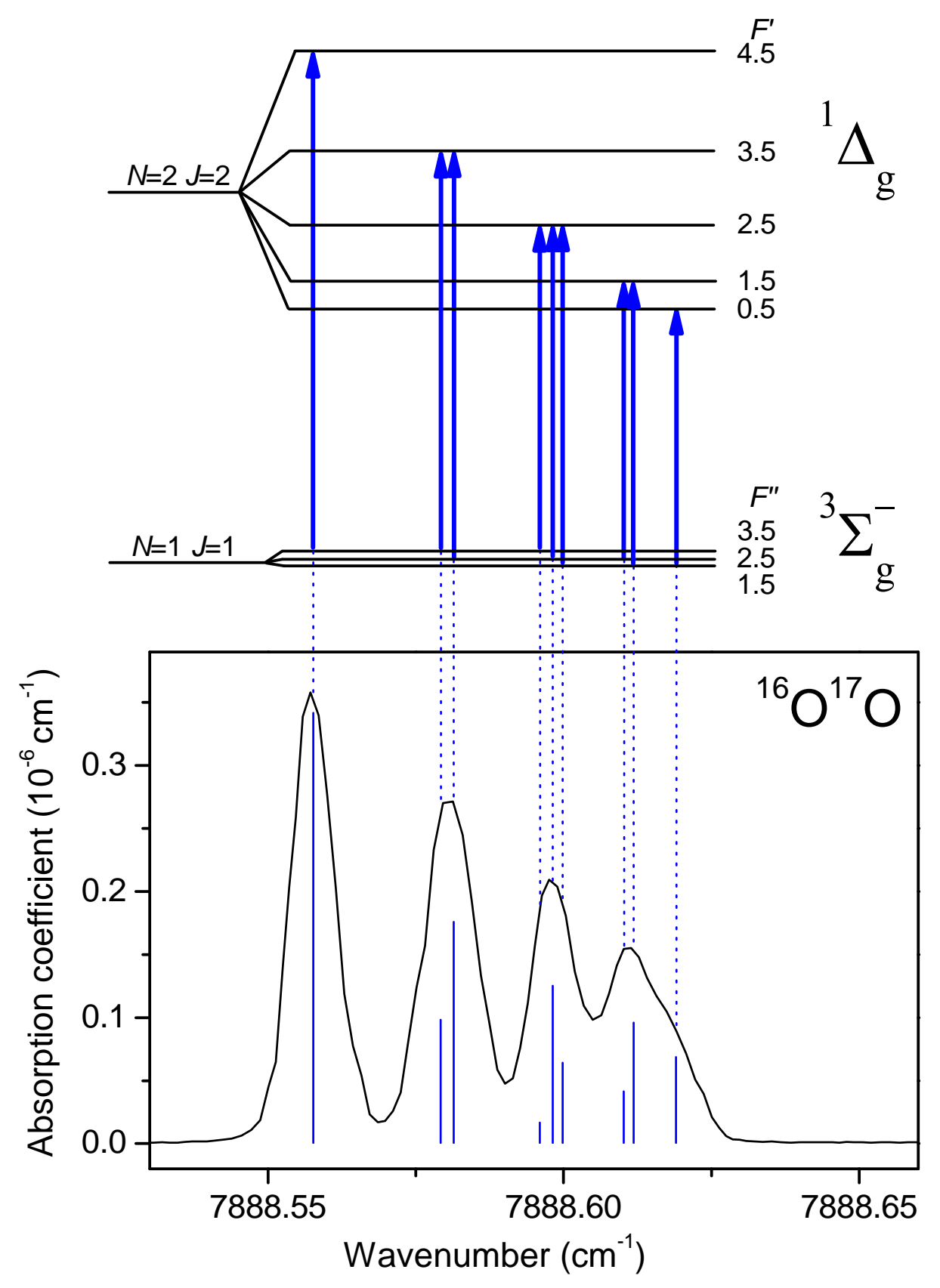


1

2

3

\section{Hyperfine structure of the $a^{1} \Delta_{\mathrm{g}}-X^{3} \Sigma_{\mathrm{g}}^{-}$transitions of ${ }^{16} \mathrm{O}^{17} \mathrm{O},{ }^{17} \mathrm{O}^{18} \mathrm{O}$ and ${ }^{17} \mathrm{O}_{2}$ by CRDS at $80 \mathrm{~K}$}

*Corresponding author: Tel.: +334 765143 19; Fax: +334 76635495.

E-mail address: Alain.CAMPARGUE@ujf-grenoble.fr (Alain CAMPARGUE).

Keywords: Oxygen, $\mathrm{O}_{2}$, hyperfine structure, Cavity ring down spectroscopy, cryogenic cell 


\begin{abstract}
The high sensitivity absorption spectrum of the $a^{1} \Delta_{\mathrm{g}}-X^{3} \Sigma_{\mathrm{g}}^{-}$band of ${ }^{16} \mathrm{O}^{17} \mathrm{O},{ }^{17} \mathrm{O}^{18} \mathrm{O}$ and ${ }^{17} \mathrm{O}_{2}$ has been recorded by CW-Cavity Ring Down Spectroscopy near $1.27 \mu \mathrm{m}$. The spectra were obtained between 7640 and $7917 \mathrm{~cm}^{-1}$ with a ${ }^{17} \mathrm{O}$-enriched sample at room temperature and at 80 $\mathrm{K}$. Due to the $I=5 / 2$ nuclear spin of the ${ }^{17} \mathrm{O}$ atom, the nuclear hyperfine structure of the transitions could be partly resolved at low temperature. The parameter coupling of the nuclear spin to the electronic angular momentum in the $a^{1} \Delta_{\mathrm{g}}$ state has been derived for the three studied isotopologues of oxygen.
\end{abstract}




\section{Introduction}

Recent CRDS experiments carried out at the Grenoble University have allowed significant improvement of our knowledge of the spectral line parameters of the $a^{1} \Delta_{\mathrm{g}}-X^{3} \Sigma_{\mathrm{g}}^{-}$band of oxygen. In Ref [1], the electric quadrupole line parameters were found to have sufficient intensities to be detected in the atmospheric spectrum and they were parameterized to complement magnetic dipole transitions provided in HITRAN database [2]. In Ref. [3], the measurements in ${ }^{16} \mathrm{O}$ - and ${ }^{18} \mathrm{O}$ - enriched mixtures of oxygen have allowed determination of the best to date spectroscopic constants in the $a^{1} \Delta_{\mathrm{g}}$ band for five most abundant isotopologues of oxygen $\left({ }^{16} \mathrm{O}_{2},{ }^{16} \mathrm{O}^{18} \mathrm{O},{ }^{18} \mathrm{O}_{2},{ }^{16} \mathrm{O}^{17} \mathrm{O}\right.$ and $\left.{ }^{17} \mathrm{O}^{18} \mathrm{O}\right)$. For the ${ }^{17} \mathrm{O}$-containing species significant broadening of rotational lines due to unresolved hyperfine structure was observed [3].

Indeed, unlike other isotopes of oxygen ${ }^{17} \mathrm{O}$ has a non-zero nuclear spin $(I=5 / 2)$ and the coupling of the nuclear spin to electron spin in the $X^{3} \Sigma_{\mathrm{g}}^{-}$state [3] and to the electronic angular momentum in the $a^{1} \Delta_{\mathrm{g}}$ state [4] gives rise to a magnetic hyperfine (hf) structure. As a result of the Doppler broadening at room temperature, the magnetic hyperfine structure could not be resolved and it manifested itself only as a broadening of the ${ }^{16} \mathrm{O}^{17} \mathrm{O}$ and ${ }^{17} \mathrm{O}^{18} \mathrm{O}$ transitions.

The present study is devoted to a detailed experimental investigation of the hf structure of the ${ }^{17} \mathrm{O}$ containing isotopologues of oxygen, on the basis of new CRDS spectra of the $a^{1} \Delta_{\mathrm{g}}-X$ ${ }^{3} \Sigma_{\mathrm{g}}^{-}$band recorded at room temperature and at liquid nitrogen temperature (about $80 \mathrm{~K}$, see below). By using a ${ }^{17} \mathrm{O}$-enriched sample, the ${ }^{16} \mathrm{O}^{17} \mathrm{O},{ }^{17} \mathrm{O}^{18} \mathrm{O}$ and ${ }^{17} \mathrm{O}_{2}$ isotopologues could be simultaneously studied. As illustrated below, the narrowing by a factor of two of the Doppler width at $80 \mathrm{~K}$ compared to room temperature was decisive to (partly) resolve and interpret the hf structure.

After the description of the CRDS spectrometer in the next section, the obtained spectra and the analysis of the hf structure will be presented in Section 3. The coupling parameters will be determined and compared to the values available in the literature [4].

\section{Experimental}

\section{Room temperature measurements}

The high-sensitivity CW-CRDS absorption spectrum of the $a^{1} \Delta_{\mathrm{g}}-X^{3} \Sigma_{\mathrm{g}}^{-}$band was recorded at room temperature (RT) in the $7658-7917 \mathrm{~cm}^{-1}$ region using a ${ }^{17} \mathrm{O}$-enriched sample. The stated 
atomic composition of the sample (from Sigma Aldrich) was ${ }^{17} \mathrm{O}: 55.8 \%,{ }^{16} \mathrm{O}: 43.4 \%$ and ${ }^{18} \mathrm{O}$ : $0.8 \%$.

The fibered distributed feedback (DFB) laser CW-CRDS spectrometer used for the recordings at room temperature is the same as the one used for the study of the natural and ${ }^{18} \mathrm{O}$ enriched samples [1, 3]. It has been described in details in Refs. [6-8]. Each DFB laser diode has a typical tuning range of about $40 \mathrm{~cm}^{-1}$ by temperature tuning from $-10^{\circ} \mathrm{C}$ to $60^{\circ} \mathrm{C}$. A total of nine DFB laser diodes were needed to cover the $7658-7917 \mathrm{~cm}^{-1}$ region. The electro-polished stainless steel ring down cell $(l=1.42 \mathrm{~m}$, inner diameter $\Phi=11 \mathrm{~mm})$ was fitted by a pair of super-mirrors. The reflectivity of these mirrors (about $99.997 \%$ ) corresponds to empty cell ring down times of about $\tau \sim 200 \mu \mathrm{s}$. About 40 ring down events were averaged for each spectral data point; the complete temperature scan of one DFB laser (15000 spectral points) required about 70 minutes. The achieved noise equivalent absorption was about $\alpha_{\min } \sim 4 \times 10^{-11} \mathrm{~cm}^{-1}$ over the whole spectrum. The pressure measured by a capacitance gauge (MKS 100 Torr full range with $0.1 \%$ accuracy) and the ring down cell temperature were monitored during the recordings. The pressure value of the RT spectra was fixed to 30.0 Torr. Additional recordings were performed at 5 Torr in the $7839-7917 \mathrm{~cm}^{-1}$ region corresponding to the strongest transitions.

\section{Measurements at low temperature}

The cryogenic cell used for the CW-CRDS recordings at liquid nitrogen temperature (LNT) has been described in Refs. [9-12]. It was developed and extensively used to characterize the absorption spectrum of methane at low temperature in the 1.58 and $1.28 \mu \mathrm{m}$ methane transparency windows [9-12]. Briefly, it is based on an original design (see Fig. 1 of Ref. [9]) which dispenses with an external vacuum jacket by exploiting the fact that a low pressure gas sample constitutes itself a good thermal insulation. The cryostat is a $1.42 \mathrm{~m}$ long hollow cylinder both filled and completely surrounded by the sample gas volume. The liquid nitrogen filling tube constitutes its single suspension point which, combined with an almost perfect cylindrical geometry helps to eliminate stress normally present in a double-jacket cell configuration. Contrary to our previous setup [9], one of the mirrors was prealigned then glued on a piezo actuator allowing for cavity length modulation. Therefore a standard CW-CRDS data acquisition could be used with a spectral calibration fully relying on the wavemeter real time measurements. The distance between the high reflective mirrors and the cryostat ends is about $0.5 \mathrm{~cm}$. A sensitivity comparable to that achieved at room temperature $\left(\alpha_{\min } \sim 10^{-10} \mathrm{~cm}^{-1}\right)$ was obtained. 
Coinciding temperature values $(80 \pm 2 \mathrm{~K})$ were obtained from the Doppler profile of methane absorption lines $[9,10]$ and the rotational intensity distribution of the $3 v_{2}$ band of $\mathrm{CH}_{3} \mathrm{D}$ [12]. This value of $80 \pm 2 \mathrm{~K}$ is what we refer to as "liquid nitrogen temperature" (LNT).

The LNT spectrum was recorded in the $7876-7893 \mathrm{~cm}^{-1}$ region corresponding to the low rotational transitions which exhibit the most resolved hyperfine structures of the ${ }^{17} \mathrm{O}$ containing isotopologues. The pressure value was fixed to 1.5 Torr.

Fig. 1 shows the different line profiles obtained for the R1R1 transitions of the ${ }^{16} \mathrm{O}_{2},{ }^{16} \mathrm{O}^{17} \mathrm{O}$ and ${ }^{17} \mathrm{O}_{2}$ isotopologues (Note that we use $\Delta N N^{\prime \prime} \Delta J J^{\prime \prime}$ notation where $N$ and $J$ are the quantum numbers relative to the rotational angular momentum and total angular momentum, respectively). While the ${ }^{16} \mathrm{O}_{2}$ line appears as single line with a Doppler limited profile, the ${ }^{16} \mathrm{O}^{17} \mathrm{O}$ and ${ }^{17} \mathrm{O}_{2}$ lines are split in different hf components. The comparison between the $296 \mathrm{~K}$ and $80 \mathrm{~K}$ spectra (Fig.1) illustrates the much better resolution of the hf structure at $80 \mathrm{~K}$ due to the reduction by a factor of two of the Doppler broadening (HWHM of 0.008 and $0.004 \mathrm{~cm}^{-1}$ at $296 \mathrm{~K}$ and $80 \mathrm{~K}$, respectively).

\section{Spectrum calibration}

Each $40 \mathrm{~cm}^{-1}$ wide spectrum recorded with one DFB laser was calibrated independently on the basis of the wavelength values provided by a Michelson-type wavemeter (Bristol 621A, 60 $\mathrm{MHz}$ resolution and $100 \mathrm{MHz}$ accuracy). The calibration was further refined by stretching the whole spectrum in order to match accurate positions of the transitions of the $a^{1} \Delta_{\mathrm{g}}-X^{3} \Sigma_{\mathrm{g}}^{-}$band of ${ }^{16} \mathrm{O}_{2}$ provided in the recent HITRAN update [2]. The uncertainty in the absolute values of the line positions is estimated to be $1 \times 10^{-3} \mathrm{~cm}^{-1}$. It results from an equivalent contribution of the uncertainties in the HITRAN values and in the values measured in the present study.

\section{Results}

Transitions due to the ${ }^{17} \mathrm{O}_{2},{ }^{16} \mathrm{O}^{17} \mathrm{O},{ }^{16} \mathrm{O}_{2},{ }^{17} \mathrm{O}^{18} \mathrm{O}$ and ${ }^{16} \mathrm{O}^{18} \mathrm{O}$ isotopologues were found to contribute to the spectrum. The most abundant species are ${ }^{17} \mathrm{O}_{2},{ }^{16} \mathrm{O}^{17} \mathrm{O}$ and ${ }^{16} \mathrm{O}_{2}$ with relative concentration on the order of $0.33,0.47$ and 0.18 , respectively. Overall, more than one thousand transitions were measured in the RT spectrum. The global rovibrational analysis including the complete line list, description of the assignment procedure and derivation of the spectroscopic parameters from a global fit will be published in a separate contribution. In the present Letter, we limit the discussion to the hf structure of the ${ }^{17} \mathrm{O}_{2},{ }^{16} \mathrm{O}^{17} \mathrm{O}$ and ${ }^{17} \mathrm{O}^{18} \mathrm{O}$ species. 
While ${ }^{16} \mathrm{O}$ and ${ }^{18} \mathrm{O}$ nuclei have a $I=0$ nuclear spin, ${ }^{17} \mathrm{O}$ has a $I=5 / 2$ spin leading to the existence of an hyperfine structure in the ${ }^{17} \mathrm{O}$ containing species. The hf structure affecting the $X$ ${ }^{3} \Sigma_{\mathrm{g}}^{-}$ground state of ${ }^{17} \mathrm{O}_{2},{ }^{16} \mathrm{O}^{17} \mathrm{O}$ and ${ }^{17} \mathrm{O}^{18} \mathrm{O}$ was previously reported from microwave and millimeter spectroscopy measurements [5, 13-16]. The observation of the ground state hyperfine structure by absorption spectroscopy in the near infrared or visible is particularly difficult as the Doppler width is significantly larger than the hf splitting. Very recently, Long et al. [14] could measure the ground hyperfine structure from an excess of the broadening of some transitions of the $b^{1} \Sigma_{\mathrm{g}}{ }^{+}-X^{3} \Sigma_{\mathrm{g}}^{-}$band near $760 \mathrm{~nm}$. The sub Doppler hyperfine structure could be evidenced from a detailed study of the line profile recorded by Frequency-stabilized CRDS [14]. It is important to note that the evidenced extra broadening of the $b^{1} \Sigma_{\mathrm{g}}{ }^{+}-X^{3} \Sigma_{\mathrm{g}}^{-}$band transitions is due to the ground state hf structure as the $b^{1} \Sigma_{\mathrm{g}}{ }^{+}$upper electronic state is a singlet $\Sigma$ state with negligible hf structure. In the present study devoted to the $a^{1} \Delta_{\mathrm{g}}-X^{3} \Sigma_{\mathrm{g}}^{-}$band, the situation is different because the nuclear spin can couple to the electronic angular momentum of the $a^{1} \Delta_{\mathrm{g}}$ upper state. Our spectra (Fig. 1 for instance) show that the observed hf splitting is significantly larger than the Doppler broadening and then that the hf structure of the $a^{1} \Delta_{\mathrm{g}}$ upper state is larger than that of the ground state. This is consistent with earlier results of electron paramagnetic resonance (EPR) measurements of ${ }^{16} \mathrm{O}^{17} \mathrm{O}$ and ${ }^{17} \mathrm{O}^{18} \mathrm{O}$ species in the $a^{1} \Delta_{\mathrm{g}}$ state [4].

The general expression of the hyperfine structure Hamiltonian can be written as

$$
\mathbf{H}_{h f}=a \mathbf{I}_{z} \mathbf{L}_{z}+b_{F} \mathbf{I} \cdot \mathbf{S}+c\left(\mathbf{I}_{z} \mathbf{S}_{z}-\frac{1}{3} \mathbf{I} \cdot \mathbf{S}\right)+\frac{\mathrm{e} q Q\left(3 \mathbf{I}_{z}^{2}-\mathbf{I}^{2}\right)}{4 I(2 I-1)},
$$

where the first term describes the interaction of the electronic orbital moment with the nuclear spin, the second and third terms represent the isotropic (Fermi contact interaction) and anisotropic parts of the electron spin-nuclear spin coupling, respectively. The forth term represents the electric quadrupole interaction due to asymmetric charge distribution around the nucleus.

In the $X^{3} \Sigma_{\mathrm{g}}^{-}$ground state, $\Lambda=0$ and therefore the first term vanishes. The two other magnetic terms lead to an hf splitting of each $(N, J)$ rotational level in different components:

$$
\begin{gathered}
W\left(F_{1}\right)=\frac{\mathbf{I J}}{(N+1)}\left\{b_{F}+c \frac{2 N}{(2 N+3)}\right\} \\
W\left(F_{2}\right)=\frac{\mathbf{I J}}{N(N+1)}\left(b_{F}+\frac{2}{3} c\right)
\end{gathered}
$$




$$
W\left(F_{3}\right)=\frac{\mathbf{I} \mathbf{J}}{N}\left\{-b_{F}+c\left(\frac{2 N+2}{2 N-1}\right)\right\},
$$

where $\mathbf{I J}=1 / 2[F(F+1)-I(I+1)-J(J+1)]$ with $F=J+I, J+I-1, \ldots|J-I|$ and $F_{1}, F_{2}$ and $F_{3}$ : corresponds to $J=N+1, J=N$ and $J=N-1$, respectively. Note that Eq. 2 ignores small contribution due to quadrupole coupling.

In the case of the ${ }^{16} \mathrm{O}^{17} \mathrm{O}$ and ${ }^{17} \mathrm{O}^{18} \mathrm{O}$ species, $I=5 / 2$ and then $F=J \pm 5 / 2, J \pm 3 / 2$ and $J \pm 1 / 2$. The rotational levels are then split into six components for $J>2$.

In the case of the ${ }^{17} \mathrm{O}_{2}$ isotopologue, the vectorial addition of the two nuclear spins $\left(I_{1}=I_{2}=5 / 2\right)$ leads to six values of the total nuclear spin $I_{t o t}=I_{1}+I_{2}, \ldots\left|I_{1}-I_{2}\right|=5,4,3,2,1,0$. In the $X$ ${ }^{3} \Sigma_{\mathrm{g}}^{-}$ground state because of the nuclear spin function symmetry, the even and odd values of $N^{\prime \prime}$ can only be associated with odd and even values of $I_{t o t}$, , respectively [13]. Thus each $J^{\prime \prime}$ level is split into $F=J+I_{t o t}, J+I_{t o t}-1, \ldots\left|J-I_{t o t}\right|$ components with $I_{t o t}=5,3,1$ for even $N^{\prime \prime}$ values and with $I_{t o t}=4,2,0$ for odd $N^{\prime \prime}$ values. For instance, for $N=1$ the number of components is seven, while for $N=2$ the level splits into 13 components.

The $a^{1} \Delta_{\mathrm{g}}$ upper state is a singlet state $(S=0)$, so that only the first and fourth interaction terms remain in Eq. 1. This $a^{1} \Delta_{\mathrm{g}}$ state obeys the Hund's electronic case (a) coupling and the dominant magnetic part of the hyperfine operator simplifies to yield the hyperfine energy [17]:

$$
W=a \Lambda \Omega \mathbf{I J} / J(J+1) \text {. }
$$

with $\Omega=\Lambda+\Sigma$, which in this case is equal to $\Lambda=2$. From this expression, it is obvious that the hyperfine component separations decrease with increasing $J$ values i.e. that, in the different branches, transitions from the first rotational levels are expected to show the most resolved hf structure. As an example, Figs. 2 and 3 illustrate the evolution of the hf structure with an increasing rotational excitation for ${ }^{16} \mathrm{O}^{17} \mathrm{O}$ and ${ }^{17} \mathrm{O}_{2}$, respectively: the hf structure is partly resolved for the first $N, J$ rotational levels but shows up as a small extra broadening for $N, J$ values larger than 10. It is worth mentioning that $\Lambda$-doubling could not be resolved in this experiment.

The selection rules for ${ }^{16} \mathrm{O}^{17} \mathrm{O},{ }^{17} \mathrm{O}^{18} \mathrm{O}$ and ${ }^{17} \mathrm{O}_{2}$ magnetic dipole transitions are $\Delta J=0, \pm 1$ and $\Delta F=0, \pm 1$. For the high $J$ values, only the hyperfine transitions with $\Delta J=\Delta F$ are significant and the intensity of these transitions are proportional to $(2 F+1)$ [18]. Fig. 4 shows the energy diagram and allowed transitions of the R1R1 line together with the corresponding experimental 
spectra of ${ }^{16} \mathrm{O}^{17} \mathrm{O}$ and ${ }^{17} \mathrm{O}^{18} \mathrm{O}$. The hf coupling terms being identical for these two species, the observed hf structures are very similar.

The values of the hf coupling parameters were determined from a global fit of all the transitions measured in this work together with the microwave (MW) lines from Cazzoli et al. [5, 13] by using the SPFIT software [19]. The expression of the rotational part of the Hamiltonians in the ground and excited electronic states is given in Ref. [3] (Eqs. 1 and 2). A detailed description of the fit including the weighting of the wavenumbers values of the hf components, the combination of RT and LNT datasets, the constraints of the fits of the spectroscopic parameters and comparisons with previously reported values will be detailed in a separate contribution [20]. In this Letter, we focus on the obtained hf coupling constants which are listed in Table 1 for both the ground and $a^{1} \Delta_{\mathrm{g}}$ upper states. Note that we have tried to fit the quadrupole coupling constant of the $a^{1} \Delta_{\mathrm{g}}$ (last term of Eq. 1) but it could not be well determined. The corresponding constant in the ground state of ${ }^{16} \mathrm{O}^{17} \mathrm{O}$ and ${ }^{17} \mathrm{O}^{18} \mathrm{O}$ is about $-8 \mathrm{MHz}$ and according to the calculations of Minaev and Minaeva [21] it should be similar in the excited state and then too small to be determined from our spectra.

To the best of our knowledge, the only previous determination of the hf coupling constant in the $a^{1} \Delta$ state is due to Arrington et al. [4] who obtained the $a$ value of ${ }^{16} \mathrm{O}^{17} \mathrm{O}$ and ${ }^{17} \mathrm{O}^{18} \mathrm{O}$ from a fit of EPR spectra [4]. Since in theory there is no isotopic dependence for this parameter, they performed a global fit for the two species and determined the coupling constant to be $-424 \pm 1$ $\mathrm{MHz}$ which is almost exactly a factor of two greater than our values $(-215.6 \pm 2.3$ and $-212.4 \pm 3.5$ $\mathrm{MHz}$, for ${ }^{16} \mathrm{O}^{17} \mathrm{O}$ and ${ }^{17} \mathrm{O}^{18} \mathrm{O}$, respectively). This difference is merely due to the different definition of the coupling constant adopted by Arrington et al. [4], the value of $\Lambda(\Lambda=2$ in this case) being absorbed into the constant. The hf coupling parameter of ${ }^{17} \mathrm{O}_{2}$ could be also determined from the analysis of our LNT spectrum. As expected, the obtained value is very close to that of the ${ }^{16} \mathrm{O}^{17} \mathrm{O}$ and ${ }^{17} \mathrm{O}^{18} \mathrm{O}$ isotopologues.

We have superimposed to the spectra of Figs. 2 and 3 a simulation of the hf structure calculated with the fitted parameters values. The simulated spectra were obtained by affecting a Doppler limited line profile (at $80 \mathrm{~K}$ ) to the stick spectrum provided by the SPFIT program. It leads to a very convincing agreement between the measured and calculated spectra. 


\section{Conclusion}

The combination of the high sensitivity CRDS technique with a cryogenic cell at $80 \mathrm{~K}$ has allowed for the first observation of the partly resolved hyperfine structure of the $a^{1} \Delta_{\mathrm{g}}-X^{3} \Sigma_{\mathrm{g}}^{-}$band of the three ${ }^{17} \mathrm{O}$ containing isotopologues of oxygen $-{ }^{16} \mathrm{O}{ }^{17} \mathrm{O},{ }^{17} \mathrm{O}^{18} \mathrm{O}$ and ${ }^{17} \mathrm{O}_{2}$. The observed structure results from the hf splittings of both the $X^{3} \Sigma_{\mathrm{g}}^{-}$ground state and the $a^{1} \Delta_{\mathrm{g}}$ upper state which have a different origin: the hf splitting of the $X^{3} \Sigma_{\mathrm{g}}^{-}$levels is due to the coupling of the ${ }^{17} \mathrm{O}$ nuclear spin to the electronic spin while that of the $a^{1} \Delta_{\mathrm{g}}$ upper states is larger and results from the coupling of the ${ }^{17} \mathrm{O}$ nuclear spin to the electronic orbital angular momentum. The hf coupling parameters in the excited state have been determined from a global fit of the line positions. The simulation of the hf structure calculated using these parameters leads to a very satisfactory reproduction of the measured hf structure.

\section{Acknowledgments}

Part of this work was performed at Grenoble University under the ANR project "IDEO" (NT09_436466). A portion of this research was performed at Jet Propulsion Laboratory, California Institute of Technology, under contract with the National Aeronautics and Space Administration. 


\section{References}

[1] I.E. Gordon, S. Kassi, A. Campargue, G. C. Toon J. Quant. Spectrosc. Radiat. Transfer 111 (2010) 1174.

[2] L.S. Rothman, I.E. Gordon, A. Barbe, D.C. Benner, P.F. Bernath, M. Birk et al., J. Quant. Spectrosc. Radiat. Transfer 110 (2009) 533.

[3] O. Leshchishina, S.Kassi, I.E. Gordon, L. S. Rothman, L. Wang, A. Campargue, J. Quant. Spectrosc. Radiat. Transfer 111 (2010) 2236.

[4] C. A. Arrington, A. M. Falick, R. J. Myers, J. Chem. Phys. 55, (1971) 909.

[5] G. Cazzoli, C D. Esposti, PG Favero, G Severi., Nuovo Cimento B 62 (1981) 243.

[6] P. Macko, D. Romanini, S.N. Mikhailenko, O.V. Naumenko, S. Kassi, A. Jenouvrier, et al., J. Mol. Spectrosc. 227 (2004) 90.

[7] J. Morville, D. Romanini, A.A. Kachanov, M. Chenevier, Appl. Phys. B 78 (2004) 465.

[8] B.V. Perevalov, S. Kassi, D. Romanini, V.I. Perevalov, S.A. Tashkun, A. Campargue, J. Mol. Spectrosc. 238 (2006) 241.

[9] S. Kassi, B. Gao, D. Romanini, A. Campargue, Phys. Chem. Chem. Phys. 10 (2008) 4410.

[10] S. Kassi, D. Romanini, A. Campargue, Chem. Phys. Lett. 477 (2009) 17.

[11] L. Wang, S. Kassi, A. Liu, S. Hu, A. Campargue, J. Quant. Spectrosc. Radiat. Transfer submitted

[12] L. Wang, S. Kassi, A. Liu, S. Hu, A.Campargue, J. Mol. Spectrosc. 261 (2010) 41.

[13] G Cazzoli, C D. Esposti, B. M. Landsberg, Nuovo Cimento D 3 (1984) 341.

[14] D. A. Long, D. K. Havey, M. Okumura, C. E. Miller, J. T. Hodges, Phys. Rev. 81 (2010) 064502.

[15] S. L. Miller and C. H. Townes, Phys. Rev. 90 (1953) 537.

[16] P. Gerber, Helv. Phys. Acta 45 (1972) 655.

[17] R.A. Frosch, H.M. Foley, Phys. Rev. 88 (1952) 1337.

[18] C. H. Townes and A. K. Schawlow, Microwave Spectroscopy (Dover, New York, 1975).

[19] Pickett HM, J Mol. Spectrosc. 148 (1991) 371.

[20] O. Leshchishina, S. Kassi, I.E. Gordon, S. Yu, A. Campargue I. Quant. Spectrosc. Radiat. Transfer, submitted

[21] B. F. Minaev, V.A. Minaeva, Phys. Chem. Chem. Phys. 3 (2001) 720. 
Table 1.

The hf coupling parameters of the $X^{3} \Sigma_{\mathrm{g}}^{-}$and $\mathrm{a}^{1} \Delta_{g}$ states of ${ }^{16} \mathrm{O}^{17} \mathrm{O},{ }^{17} \mathrm{O}^{18} \mathrm{O}$ and ${ }^{17} \mathrm{O}_{2}$.

\begin{tabular}{|c|c|c|c|c|}
\cline { 2 - 5 } \multicolumn{1}{c|}{} & Parameter $(\mathrm{MHz})$ & ${ }^{16} \mathrm{O}^{17} \mathrm{O}$ & ${ }^{17} \mathrm{O}^{18} \mathrm{O}$ & ${ }^{17} \mathrm{O}_{2}$ \\
\hline \multirow{2}{*}{$a^{1} \Delta_{\mathrm{g}}$} & $a$ (This work) & $-217.33(195)$ & $-211.50(261)$ & $-211.93(126)$ \\
& $a[4]$ & $-424(1)^{\mathrm{a}}$ & $-424(1)^{\mathrm{a}}$ & \\
\hline \multirow{3}{*}{${ }^{3} \Sigma_{\mathrm{g}}^{-}$} & $b_{F}$ & $-54.7444(92)$ & $-54.745(21)$ & $-54.758(23)$ \\
& $c$ & $140.079(24)$ & $140.090(59)$ & $140.0378(62)$ \\
& $e Q q$ & $-8.13(24)$ & $-8.23(47)$ & $-8.307(40)$ \\
\hline
\end{tabular}

a) In Ref [4], there is a factor of 2 in the $a$ parameter due to a different definition. 


\section{Fig.1}

\section{Figure Captions}

The RIRl transition of ${ }^{16} \mathrm{O}_{2},{ }^{16} \mathrm{O}^{17} \mathrm{O}$ and ${ }^{17} \mathrm{O}_{2}$ at $296 \mathrm{~K}$ and $80 \mathrm{~K}$. Spectra recorded at the room $(P=5.0$ Torr $)$ and liquid nitrogen temperature $(P=1.5$ Torr $)$ are shown on the upper and lower panel, respectively.

Fig. 2

The hyperfine structure of different ${ }^{16} \mathrm{O}^{17} \mathrm{O}$ transitions ( $T=80 \mathrm{~K}, \mathrm{P}=1.5 \mathrm{Torr}$ ). The wavenumber values $\left(v_{0}\right)$ corresponding to the centre of the spectral range of each panel are the following: RIR1 - 7888.5906, R2R2 - 7891.2606, R1Q2 - 7890.4704, Q6Q6 - 7882.3106 and Q13Q13 $7879.6066 \mathrm{~cm}^{-1}$.

The black solid line corresponds to the experimental spectrum while the red dash line is a simulation based on the calculated stick spectrum (in blue).

Fig. 3

The hyperfine structure of different ${ }^{17} \mathrm{O}_{2}$ transitions ( $\mathrm{T}=80 \mathrm{~K}, \mathrm{P}=1.5$ Torr). The wavenumber values $\left(v_{0}\right)$ corresponding to the centre of the spectral range for each panel are the following: R1R1 - 7889.1152, R2R2 7891.7151, Q8R7 - 7884.4304 and Q13Q13 - $7880.4153 \mathrm{~cm}^{-1}$. On the two upper panels the features at the left hand side are ${ }^{16} \mathrm{O}^{18} \mathrm{O}$ transitions.

The black solid line corresponds to the experimental spectrum while the red dash line is a simulation based on the calculated stick spectrum (in blue).

\section{Fig. 4}

Energy diagram and spectrum of the hyperfine structure of the R1R1 transition of ${ }^{16} \mathrm{O}^{17} \mathrm{O}$ and ${ }^{17} \mathrm{O}^{18} \mathrm{O}$ in the spectrum recorded at $80 \mathrm{~K}$. Note that the $\Lambda$-doubling is unresolved in the present experiment. The stick spectrum corresponds to the calculated hf structure. 
Figure 1

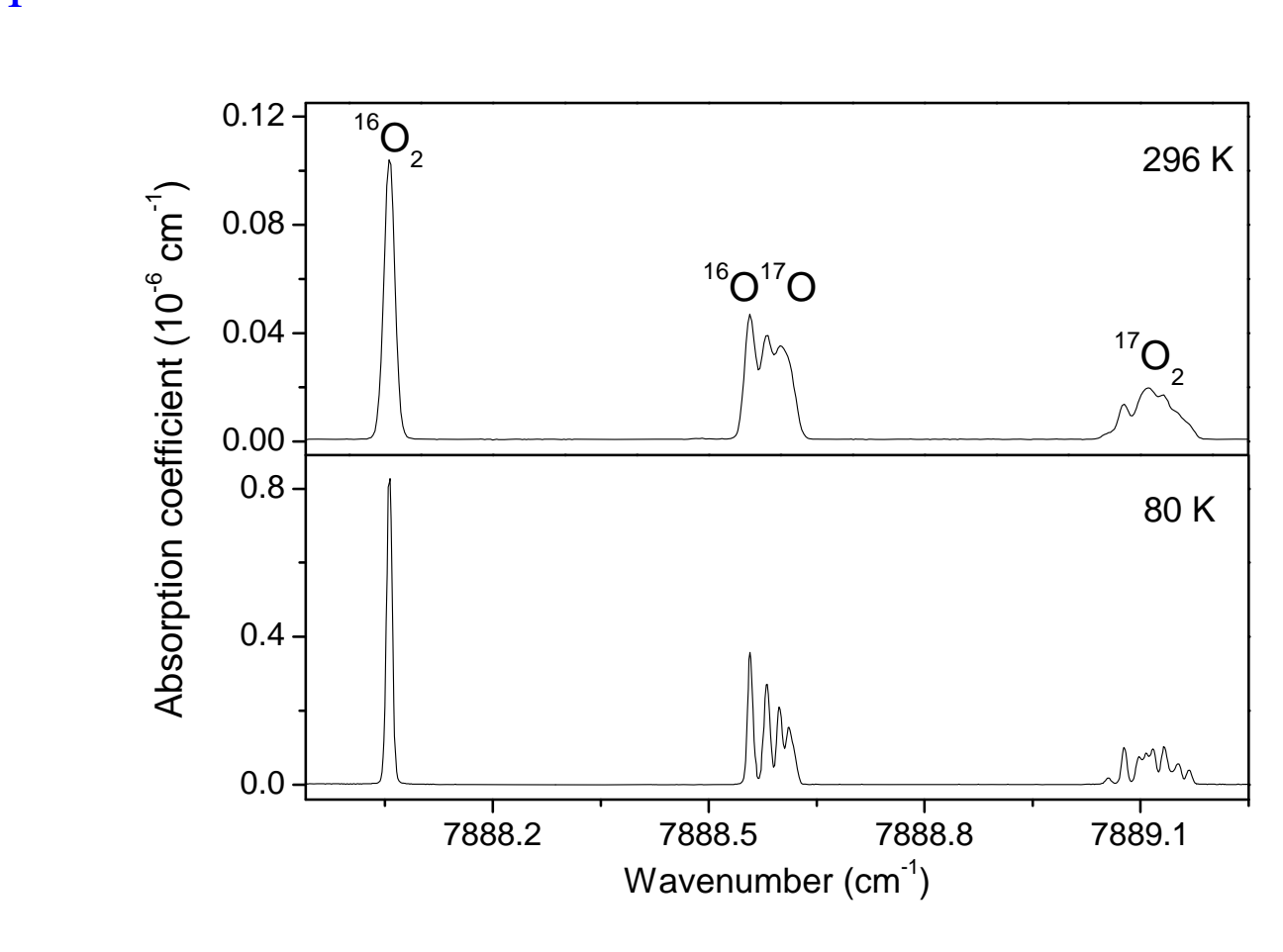

Figure

Figure 1

Figure

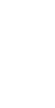
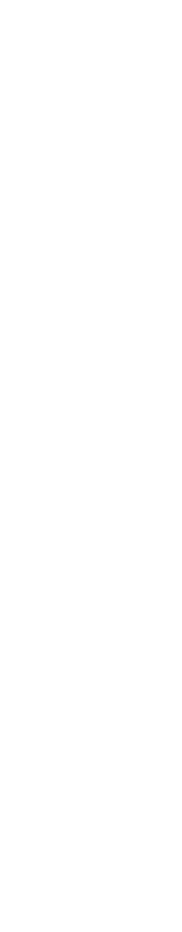

Figure

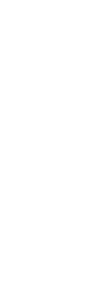

Chns

(2)

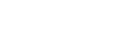


Figure 2

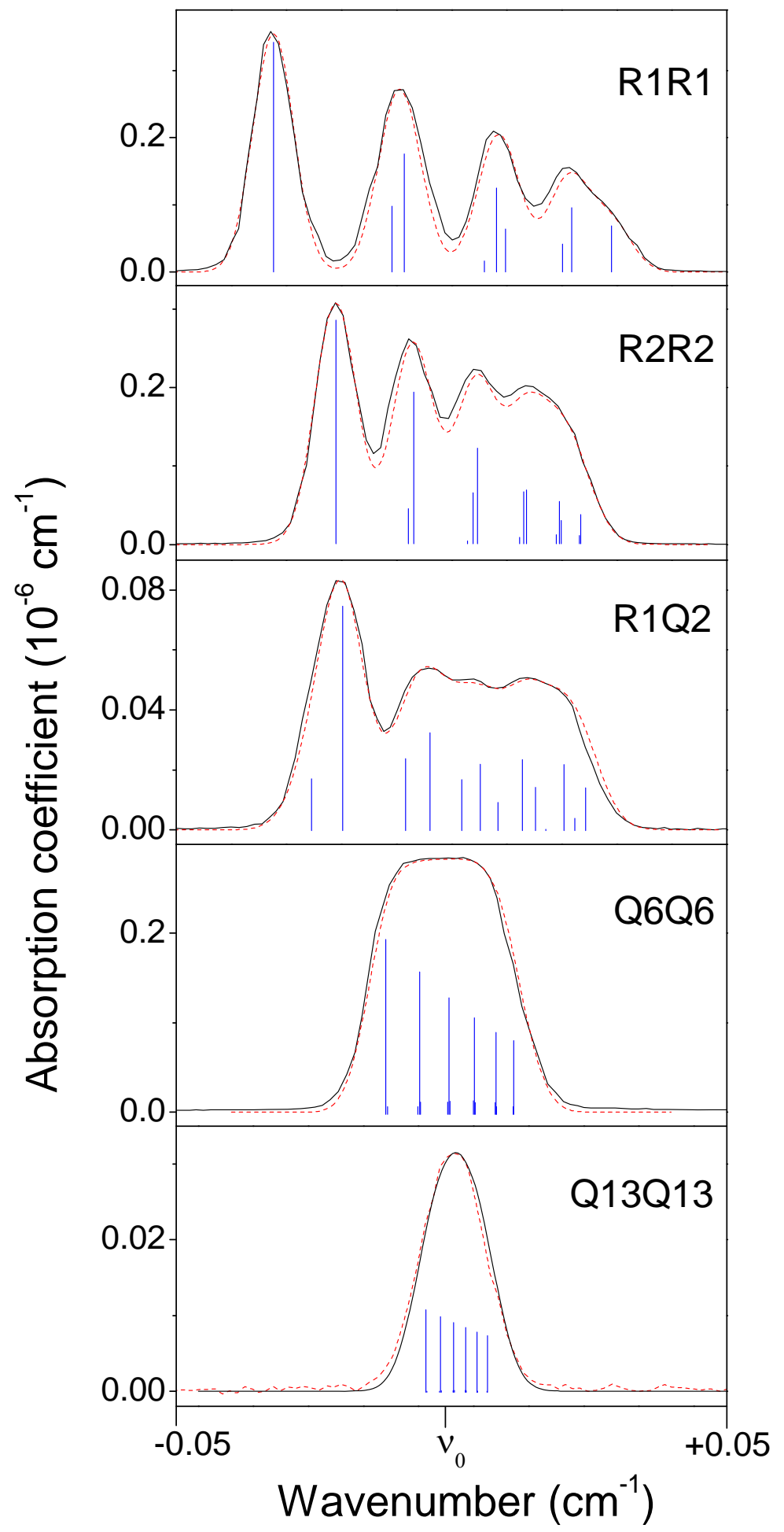


Figure 3

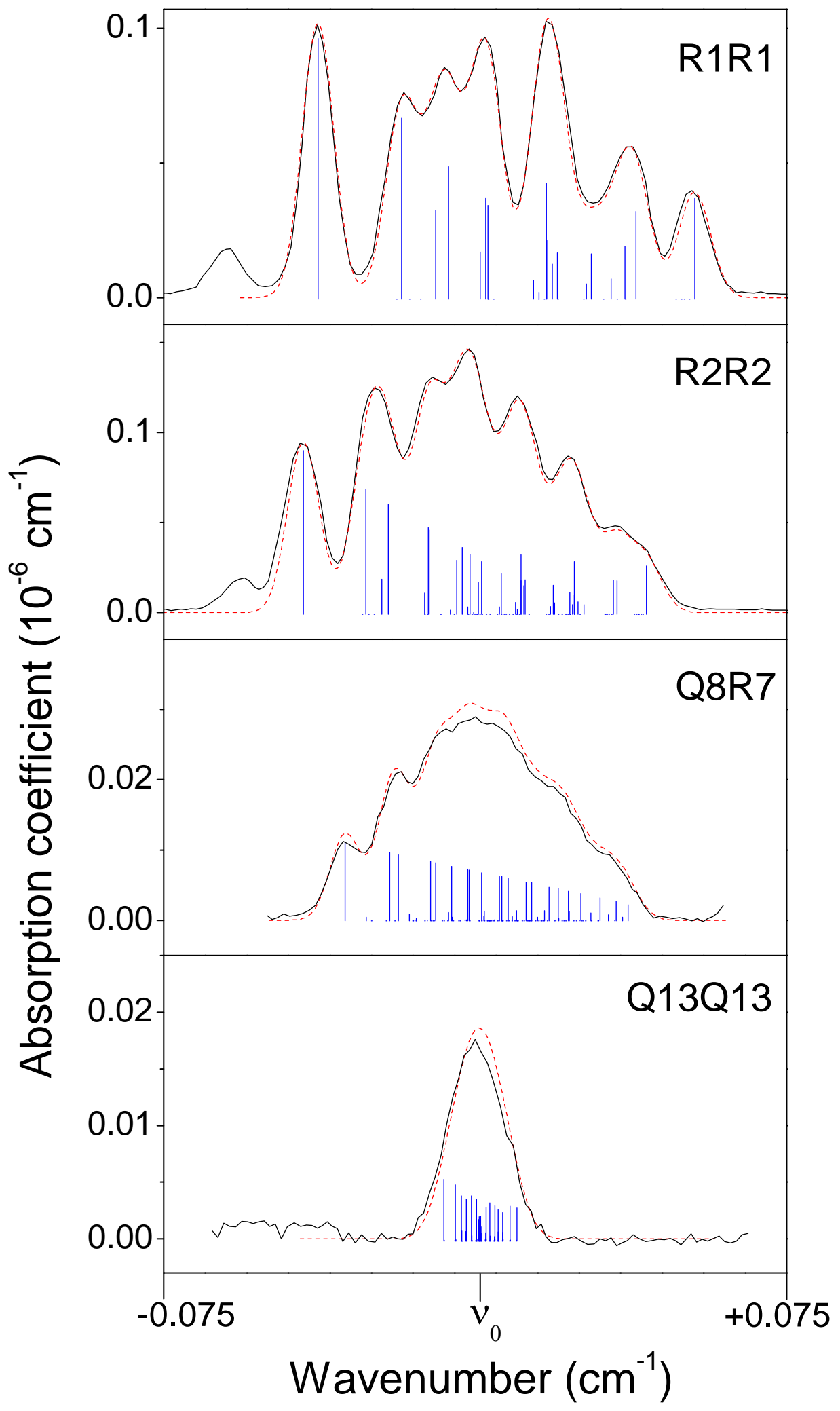


Figure 4

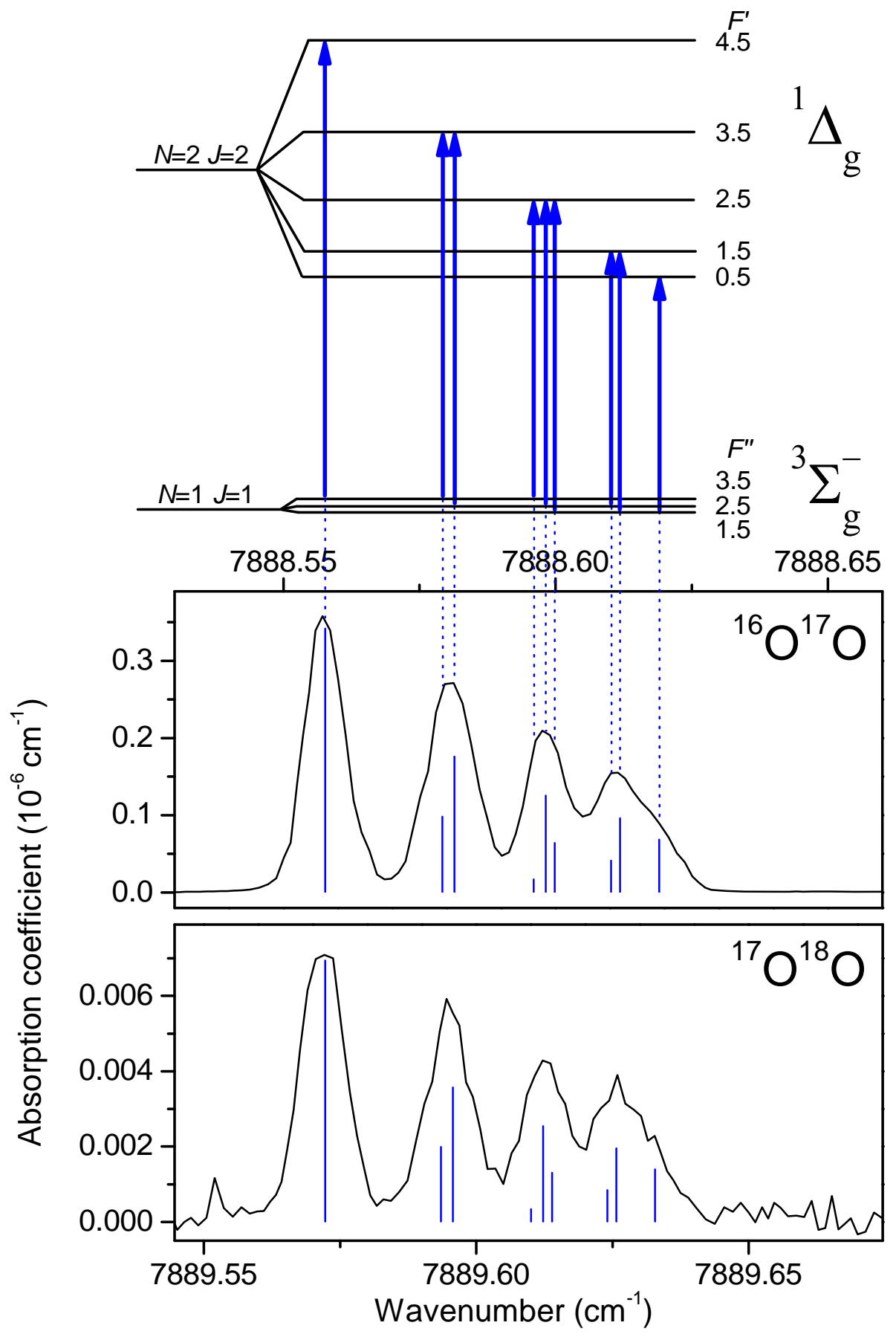

